\title{
The 2011 break in the part-time indicator and the evolution of wage inequality in Germany
}

Bernd Fitzenberger ${ }^{1,3^{*}}$ (1) and Arnim Seidlitz ${ }^{2 \dagger}$

\begin{abstract}
German social security records involve an indicator for part-time or full-time work. In 2011, the reporting procedure was changed suggesting that a fraction of worker recorded to be working full-time before the change were in fact part-time workers. This study develops a correction based on estimating the probability of being a part-time worker before and after the break. Using the correction, the paper confirms that the rise in wage inequality among full-time workers in West Germany until 2010 is not a spurious consequence of the misreporting of working time.
\end{abstract}

Keywords: Part-time indicator, SIAB7514, Wage inequality, Germany

JEL Classification: J60, J31, C80

\section{Introduction}

The Sample of Integrated Labor Market Biographies (SIAB) for the time period 1975 to 2014-and its earlier versions or larger versions of the same data-are widely used datasets for empirical analyses on the German labor market (e.g. Dustmann et al. 2009; Card et al. 2013; Möller 2016; Antonczyk et al. 2018; Biewen et al. 2018). ${ }^{1}$ The employment data in the SIAB comprise spells of employment subject to social security taxation recording in particular the length of employment, the daily gross wage, and an indicator for the part-time employment. Beyond this indicator, there is no information about hours of work. The reporting procedure for the part-time indicator changed in 2011 with dramatic consequences on the share of reported part-time workers. This paper develops a correction procedure for this break and investigates the robustness of previous findings on the evolution of wage inequality in Germany.

*Correspondence: Bernd.Fitzenberger@iab.de

${ }^{+}$Bernd Fitzenberger and Arnim Seidlitz contributed equally to this paper

${ }^{3}$ IAB (Institute for Employment Research), Regensburger Straße 100,

90478 Nuremberg, Germany

Full list of author information is available at the end of the article
Relying on the part-time indicator, the literature on longterm trends in wage inequality in Germany using SIAB data focuses on the subsample of full-time employees because of the lack of information on hours of work (see the studies cited in footnote 1). This assumes that differences in hours of work among full-time employees are negligible for the analysis of long-term trends in wage inequality thus that daily wages (earnings) provide a good approximation of the price of labor. $^{2}$ Most studies do not analyze wages for parttime employees, among whom wage differences are likely to mostly reflect differences in hours of work.

In 2011, there was a change in the reporting procedure employers had to apply for social security records (Ganzer et al. 2017; Ludsteck and Thomsen 2016; Möller

\footnotetext{
${ }^{1}$ The SIAB dataset involves a $2 \%$ sample of the Integrated Employment Biographies (IEB) of the Institute for Employment Research (IAB). The SIAB version up to 2014, denoted as SIAB7514, is described by Ganzer et al. (2017). The predecessors for earlier time periods up to 2010 were used in Dustmann et al. (2009), Antonczyk et al. (2018), and Biewen et al. (2018) -among others-for the analysis of wage inequality. Card et al. (2013) use the full population of social security records of all workers in their study on the importance of worker and firm heterogeneity for the analysis of wage inequality. The SIAB7514 was used by Möller (2016).

${ }^{2}$ Biewen and Seckler (2019) confirm the validity of this approach for an analysis of wage inequality among West German men using a different data set, namely the German Structure of Earnings Survey. The data set involves repeated cross-sections for selected years, including information for earnings and hours of work.
}

\section{Springer Open}

(c) The Author(s) 2020. This article is distributed under the terms of the Creative Commons Attribution 4.0 International License (http:// creativecommons.org/licenses/by/4.0/), which permits unrestricted use, distribution, and reproduction in any medium, provided you give appropriate credit to the original author(s) and the source, provide a link to the Creative Commons license, and indicate if changes were made. 


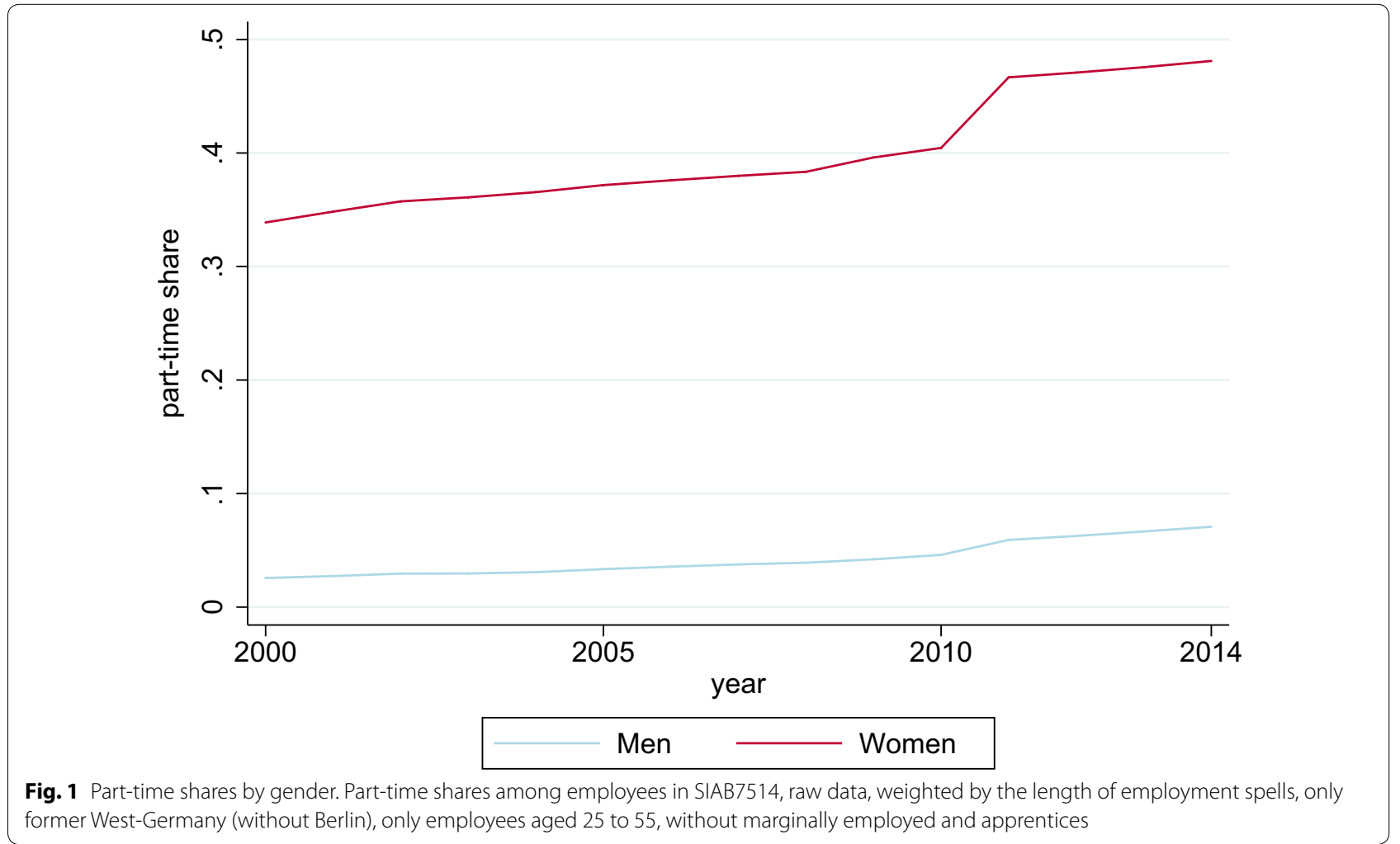

2016). Before this change, the part-time indicator was part of the occupational status variable (German: Stellung im Beruf), comprising various job characteristics. In 2011, a separate variable reporting working-time status was added (Bertat et al. 2013). ${ }^{3}$ The change both forced employers to reassess and update their reporting routines and made the need for employers to report part-time employment more salient. Thus, the reported part-time information after the change is likely to be more reliable.

The change in the reporting procedure was not implemented at the start of 2011 for all employers. There was a grace period until the end of 2011. While some employers started to use the new procedure early in 2011, others kept using the old procedure until the end of 2011. During this transition, the quality of the reported data severely deteriorated as indicated by a large number of missing values for a number of variables, including the part-time indicator. The large increase in missing values is likely to be an effect of the reporting changes. While

\footnotetext{
${ }^{3}$ The change involves the recording of the activity in the job (Tätigkeitsschlüssel), which is reported to the social security administration by the employer for each employee. It was changed from a five-digit number to be a nine-digit number (Bundesagentur für Arbeit 2019). In addition to the parttime status, it contains information on occupation, educational background, the contract period and whether employment is temporary (Bertat et al. 2013).
}

the share of missing values in the part-time indicator is below $1 \%$ in other years, it lies above $30 \%$ in the raw data in 2011. All this suggests that the new reporting procedure only started to operate fully in 2012. In this year, the number of missings in the raw data returned to normal levels.

For preparation of the most recent version of the SIAB, from 1975 to 2014 (henceforth SIAB7514), researchers at the IAB implemented an imputation for the parttime indicator in 2011 (Ganzer et al. 2017; Ludsteck and Thomsen 2016) in order to both account for the missing data and update the full data for 2011 to the new reporting procedure. Figure 1 shows that this imputation is successful insofar that the share of 2011 fits a smooth backward extrapolation of the trend for the years after 2011. At the same time, after this imputation, the change in the reporting procedure for the part-time indicator in 2011 results in a sizeable increase (fall) in the reported share of part-time (full-time) workers in 2011 as shown in Fig. 1. This structural break is larger in size for female workers (2010 to 2011 increase in part-time share by 7 percentage points, henceforth ppt), but it also affects male workers in a sizeable way (2010 to 2011 increase in part-time share by $2 \mathrm{ppt}$ ).

The jump in the level of the part-time share before and after the change in the reporting scheme is striking. 
We cannot think of a plausible reason such as a policy change, an economic shock or a mistake in the data collection from 2011 onward which could explain the sizeable increase in the part-time share. Similar in spirit to the imputation procedures applied by Ludsteck and Thomsen (2016) and Möller (2016), we argue that the higher part-time share since 2011 seems correct and therefore the lower part-time share before 2010 is likely to be the result of misreporting true part-time employment as fulltime employment. Further, there was an upward bias in reported full-time work because employers tend to reuse the record on previous employment spells by the same employees (Ludsteck and Thomsen 2016) and workers are more likely to switch from full-time work to parttime work within the same job. ${ }^{4}$ The need to update the working time status in such a case seems more salient for employers under the new reporting procedure introduced in 2011.

Möller (2016) pointed out that before 2011 full-time spells with low daily wages in the raw data are disproportionately likely to be in fact part-time spells and therefore the raw SIAB data is likely to overstate the level of wage inequality among full-timers until $2010 .^{5}$ Further, this bears the risk that the increase in wage inequality until 2010 among reported full-timers (as discussed in the literature) may also have overstated the true increase. This is an important issue because Möller's (2016) evidence based on the SIAB7514 suggests a trend reversal in wage inequality trends among full-timers in West Germany from 2011 onward, i.e. at the time of the break in the part-time indicator, such that inequality increased until 2010 and has then stopped to grow further.

Figures 2 and 3 indeed show remarkably increasing trends for different percentiles of the full-time-logearning distribution for women and men in 2011. As expected, the increase is much stronger for women which can be explained by the larger increase in the

\footnotetext{
${ }^{4}$ A legal reform in 2001 (Law on part-time and fixed-term jobs Teilzeit- und Befristungsgesetz) entitles full-time workers in Germany to switch from fulltime to part-time work in their current job. The reverse is only possible if the employer agrees.

${ }^{5}$ Frodermann et al. (2013) noted the problem earlier in the context of the return-to-job of females after motherhood when using the BASiD data set, which includes social security records for employment as does the SIAB and which also allows to identify motherhood based on merged pension data provided by the social security administration. Females who had been working full-times before motherhood were found to be recorded as working full-time after motherhood even though their daily wages had fallen strongly. The study suggests to correct employment post motherhood, which is recorded as full-time, to part-time if the daily wage was at least $10 \%$ lower than in the last employment spell before motherhood recorded as full-time by the same employer. The motivation of this correction is similar to the suggested approach by this paper. However, the details of the two correction approach differs strongly, and we do not restrict attention to mothers.
}

part-time share shown in Fig. 1. For both genders, the increase is also stronger at the bottom of the distribution. In the male distribution, one needs to consider a quite low percentile such as the 2.5 th percentile to see a large kink. This is not surprising given the low share of parttime employees among men (below 10\%). Still, the effect of the change in the reporting procedure is also visible for men-and our evidence suggests to correct employment spells up to the 25th percentile of the male full-time wage distribution. For women, a discontinuous increase from 2010 to 2011 can even be detected in the upper half of the distribution.

Möller (2016) corrects full-time employment before 2011 using a simple imputation correction, which shares some similarities with our approach. ${ }^{6} \mathrm{He}$ first estimates a non-linear trend for total part-time employment for the time period until 2010 and uses this estimate to predict part-time employment in 2011. As to be expected, this provides evidence for underreporting of part-time employment before 2011. He then fits a logit model for the incidence of part-time employment for the sample until 2010, using age, industry, wage, region among others as predictors. In the pre-2011 sample, he then corrects those reported full-time spells with the highest predicted part-time probability to part-time. This correction is continued until the break in the time trend in part-time employment, as calculated in the first step, disappears. The underlying assumption for this correction is that the relative amount of underreporting parttime employment was basically constant in the pre-2011 period.

The goal of our paper is to develop a correction based on estimating the probability of being reported as a parttime worker before and after the break in 2011. Our paper extends upon Möller (2016) in three dimensions. First, we use the year 2012 as benchmark year assuming that part-time is reported correctly in that year and we also correct wages in 2011. Second, we use an inverse probability weighting approach to reweight reported fulltime spells instead of a binary prediction as to whether a spell is full-time or part-time. Using a discrete prediction entails the danger that the correction is too strong in the bottom part of the distribution and not strong enough further up the distribution. Third, we use graphical evidence on the evolution of the wage percentiles among full-time employment to determine the position in the wage distribution below which a correction of wages is necessary.

Our approach involves estimating the probability for a part-time spell being reported among all employees

\footnotetext{
6 The details of the imputation procedure are not described in the paper and the information given here is based on personal communication.
} 

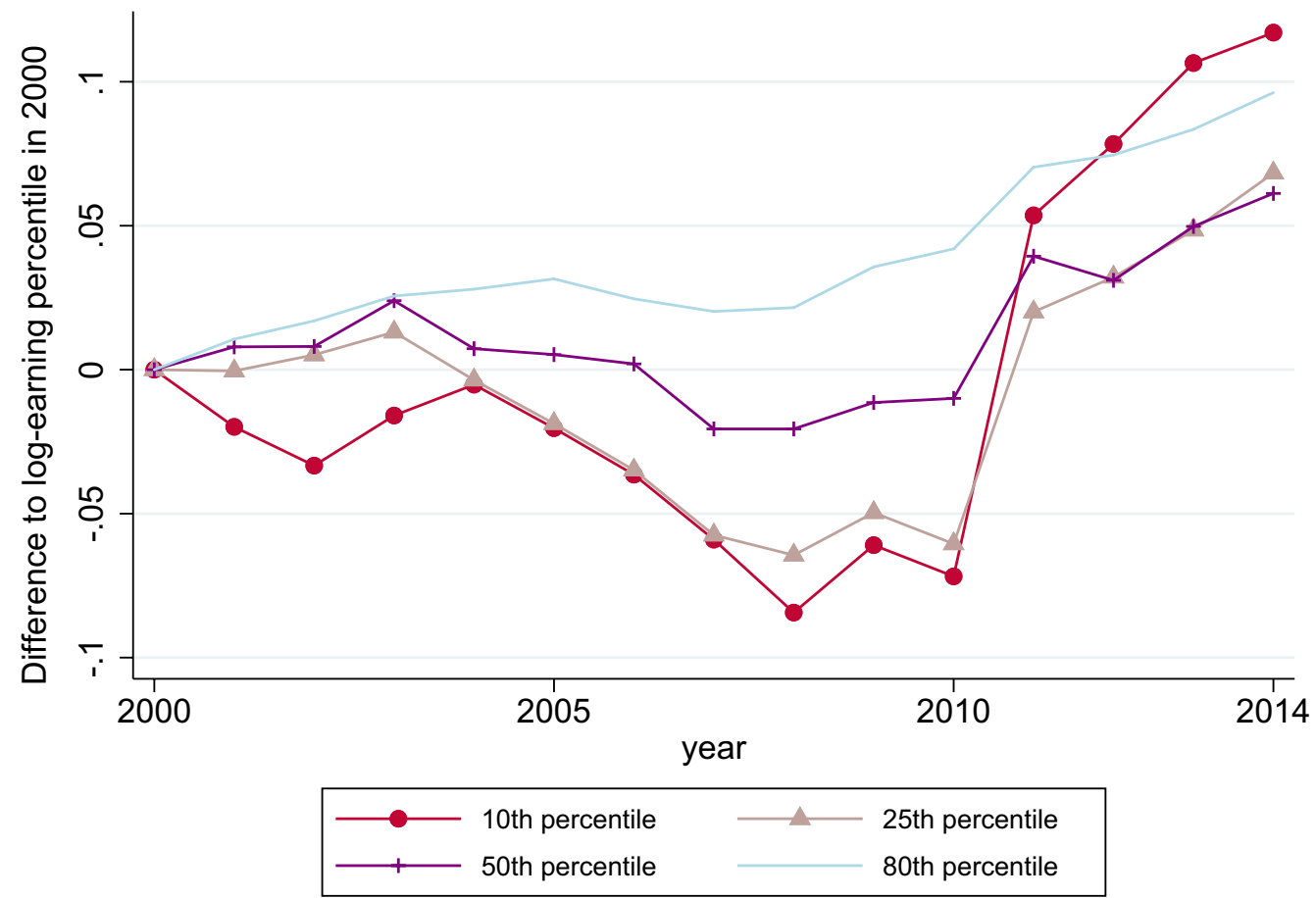

Fig. 2 Cumulative real wage growth for women, full-time, raw. Differences in log real wages over time, indexed to 0 in year 2000, only former West-Germany (without Berlin), only employees aged 25 to 55, without marginally employed and apprentices, weighted by the length of employment spells

(full-timers and part-timers) as a function of employee and job characteristics both for the year 2012 and the years before 2012. The regression is estimated based on those observations with wages below the upper bound, above which graphical evidence suggests that there is no need for correction. The rank difference in the wage distribution between the upper bound and the individual's wage is used as key covariate. Based on the regression estimates, the full-time employment data before 2012 is then reweighted using inverse probability reweighting based on the estimated propensity scores. This way, reported full-time employment spell before 2011 are corrected. We identify and downweight observations which are likely to be misreported as full-time, which results in a continuous upward correction of low wage percentiles among full-timers. This correction is smooth and we also correct the data in 2011 because our graphical evidence on wage trends suggests that the data in 2011 suffers from misreporting of low wage spells as involving full-time employment. Using our correction, the paper confirms Möller's (2016) finding that the rise in wage inequality among full-time working women in West Germany until 2010 is not a spurious consequence of the misreporting of working time. Furthermore, based on our corrected data, we find that the fall in real wages among full-timers during the 2000s was strongest among young workers [similar to the findings in Antonczyk et al. (2018) for the time period until 2004] and there is in fact a trend reversal after 2010. While real wages increase slightly after 2010 for low-wage earners among women, they stagnate at low levels or even fall further for low-wage earners among men. Wage trends between 2010 and 2014 have contributed little to reverse the strong increase in wage inequality until 2010, a findings which holds in particular for low-wage earners among men.

The remainder of this paper is organized as follows. Section 2 describes the data. Our correction approach is developed in Sect. 3. Section 4 revisits the analysis of wage inequality based on the corrected data. Section 5 concludes.

\section{Data description and sample restrictions}

In its latest version, the SIAB dataset involves a $2 \%$ sample of the Integrated Employment Biographies (IEB) of the Institute for Employment Research (IAB) from 1975 


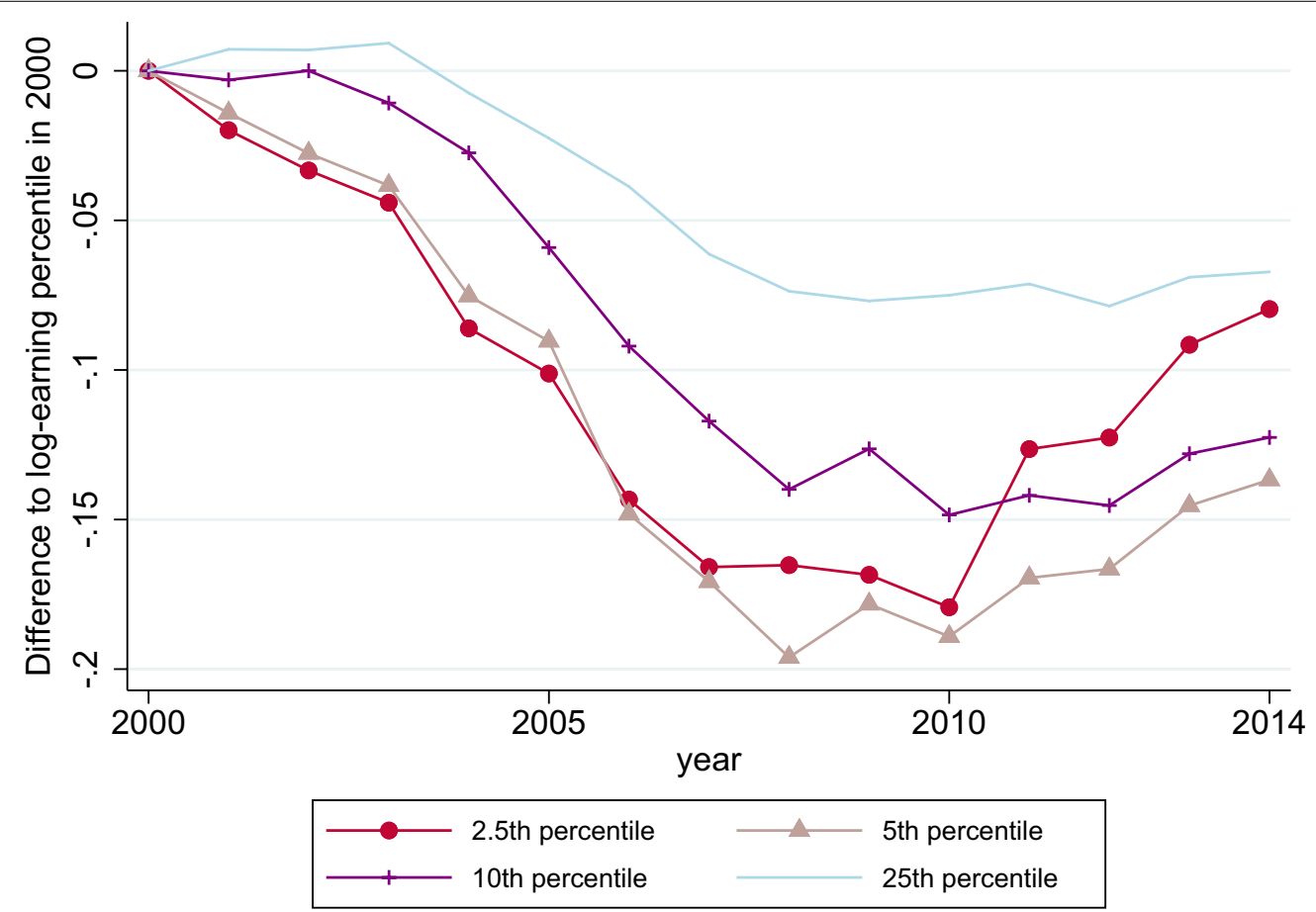

Fig. 3 Cumulative real wage growth for men, full-time, raw. Differences in log real wages over time, indexed to 0 in year 2000, only former West-Germany (without Berlin), only employees aged 25 to 55, without marginally employed and apprentices, weighted by the length of employment spells

to 2014 (for East Germany from 1992 onward). Next to data on benefit recipients, it contains information on employment spells for employees who are subject to social security contributions and marginally employed (from 1998 onward)—not included are civil servants and self-employed (Ganzer et al. 2017).

The data include the exact duration of employment spells on a daily level, some characteristics as industry, occupation, educational background, part-time status, and the daily gross-wage. The gross wage is right censored at around the 96th and 88th wage percentile for full-timers among women and men, respectively. In our empirical analysis, we analyze wage percentiles which are unaffected by the censoring. Throughout the paper all wages are given in real terms. Wages are deflated by the annual consumer price index of the Federal Statistical Office (Statistisches Bundesamt 2018). The base year for the inflation adjustment is 2014.

We apply the following sample restriction throughout the rest of our paper. We use only observations from the ten former West-German states (without Berlin) and only employees aged 25 to 55 . Additionally, we exclude employment spells for apprentices and for marginally employed. These restrictions broadly ensure that our results on wage inequality can be related to the previous literature for West Germany which used similar age restrictions (Card et al. 2013; Dustmann et al. 2009; Antonczyk et al. 2018; Biewen et al. 2018). ${ }^{7}$

Further, we weight always the employment spells by their duration in days. Employment spells are at most one year long and are always completely included in one calendar year. The maximum length of a spell is thus from January 1st to December 31st of a given year. To obtain the weight, we divide the length of all spells in days by the maximum spell duration of that year, 365 or 366 days.

\section{Correction approach for part-time indicator}

Our correction approach for the 2011 break in the parttime indicator is based on inverse probability reweighting for full-time employment spells before 2012. This is based on regressions for the probability to be reported working part-time. Based on the observation that the full-time share is generally higher in the years before 2011, we assume that there are observations which have a low probability given their characteristics to be reported as part-time in their observation year, but conditional on their characteristics their probability to be a part-time spell would be higher in 2012. Our correction approach

\footnotetext{
${ }^{7}$ Note that our approach can be applied to other age groups or to the East German states.
} 
builds on the assumption that such employment spells may be misreported in the raw data as being full-time and therefore we downweight them to reflect this possibility. In short, we estimate a correction weighting factor between zero and one for such spells. We then use these weights to reweight those spells we deem to be potentially misreported. Because of the strong gender differences in the part-time share, the correction is applied separately for women and men.

\subsection{Upper bound for correction suggested by evolution of wage percentiles}

As starting point, we argue that the observed evolution of the wage distribution before and after the structural break in 2011 suggests that there is an upper bound for wages below which a correction of the full-time status is necessary. Further, the correction below the upper bound should be the larger the lower the rank in the wage distribution, i.e. the larger the rank difference between the upper bound and the individual's wage. Figures 2 and 3 show the evolution of various uncorrected wage percentiles for men and women, respectively. Figure 3 for men shows no discontinuous upward jump for the 25th wage percentile between 2010 and 2012, a modest increase is visible at the 10th percentile and increasing jumps in absolute numbers for the 5 th and the 2.5 th percentile. Based on the graphical evidence, we take the 25th percentile of full-time wages as the upper bound for correcting the wage data for men. For women, the part-time share is much higher resulting in a stronger need for correction. Figure 2 for women shows strong increases in 2010 even for the median. At the 80th percentile, the 2010 to 2012 increase becomes rather small. Based on this evidence, we assume that wage observations above the 80th percentile of the full-time distribution for women are correctly reported and our correction applies to wages lying below this upper bound.

In the second step, we determine the rank of the upper bounds of the full-time wage distribution in the gender specific wage distribution in 2012 for total employment involving both part-timers and full-timers. ${ }^{8}$ The data reveal that the 80th and 25th percentile of the full-time distribution for women and men in 2012 correspond to the 88th and the 29th percentiles, respectively, of the total wage distribution in the same year.

As upper bounds for the corrections for the years 2000 to 2011 , we use the 88 th and 29th percentiles, respectively, in the gender specific wage distribution of total employment, assuming that there is no need for correcting full-time wages above. Using total employment, there

\footnotetext{
${ }^{8}$ Recall that we exclude marginal employment from our analysis.
}

is no risk of confusing full-time and part-time employment and the share of employment spells reported as part-time with wages above the upper bound is very small. The correction is based on reweighting full-time wages below the upper bounds based on the relative propensity of being reported as part-time in the reference year 2012 and the years before.

Formally, the role the upper bounds in the correction can be described as follows. For women, we correct all full-time wages below the year specific upper bound implied by the rank of the 80th percentile of the full-time distribution in 2012 within the distribution for total employment in the same year. Define the upper bound $\overline{\text { wage }}_{f}$ such that $F_{2012 f}\left(\overline{\text { wage }}_{f} \mid p t=0\right)=0.8$ [this is the distribution function of the full-time distribution for women in 2012, with the part-time indicator $p t=0$ ]. Then, the rank of the upper bound in the distribution for total employment is given by $F_{2012 f}\left(\overline{\text { wage }}_{f}\right)=0.88$, based on $F_{2012 f}\left[F_{2012 f}^{-1}(0.8 \mid p t=0)\right]=0.88$. We correct (downweight) spells which are reported as full-time in the years 2000 to 2011 if and only if $F_{t f}\left(\right.$ wage $\left._{t s i}\right) \leq 0.88$, where wage $_{t s i}$ denotes the wage of individual $i$ in year $t$ and spell $s$. For men, we correct analogously all full-time spells with wages below the year specific upper bound implied by the rank of the 25th percentile of the full-time distribution in 2012 within the distribution for total employment in the same year. Define $\overline{w a g e}_{m}$ such that $F_{2012 \_m}\left(\overline{\text { wage }}_{m} \mid p t=0\right)=0.25$. The rank of the upper bound in the distribution for total employment is given by $F_{2012 \_m}\left(\overline{\text { wage }}_{m}\right)=0.29$ and we correct full-time spells if $F_{t_{-} m}\left(\right.$ wage $\left._{t s i}\right) \leq 0.29$.

\subsection{Rank differences as drivers of correction}

The amount of reweighting is allowed to depend on the year specific rank difference $\theta_{t s i}$ between the wage wage $_{t s i}$ and the year specific upper bound calculated above. $\theta_{t s i}$ is calculated for the year and gender specific wage distribution for total employment. Formally, we define $\theta_{t s i}=0.88-F_{t f}\left(\right.$ wage $\left._{t s i}\right)$ for women and $\theta_{t s i}=0.29-F_{t_{-} m}\left(\right.$ wage $\left._{t s i}\right)$ for men. $\theta_{t s i}$ is zero at the upper bound and increases when moving down the wage distribution.

\subsection{Propensity score of being reported part-time in the raw data}

To estimate the part-time probability (i.e. the probability of the event complementary to being reported fulltime), we run a probit regression for reported part-time employment among all observations in full-time and part-time below the upper bound, separately by gender and year. The spells above the upper bound are not used for the regression and the full-time spells among these will later receive a weight of one. The probit regression 
Table 1 Probit regression for part-time spell reported, men

\begin{tabular}{llllll}
\hline & $\mathbf{( 1 )}$ & $\mathbf{( 2 )}$ & $\mathbf{( 3 )}$ & $\mathbf{( 4 )}$ & $\mathbf{( 5 )}$ \\
& $\mathbf{2 0 0 0}$ & $\mathbf{2 0 0 5}$ & $\mathbf{2 0 1 0}$ & $\mathbf{2 0 1 1}$ & $\mathbf{2 0 1 2}$ \\
\hline Rank diff from Upper bd. $(\theta)$ & $8.079(44.1)$ & $6.391(43.8)$ & $6.738(52.1)$ & $7.303(63.4)$ & $8.334(68.9)$ \\
Age & $0.046(4.2)$ & $0.064(6.5)$ & $0.026(3.0)$ & $0.031(3.9)$ & $0.033(4.1)$ \\
Age2 & $-0.001(3.9)$ & $-0.001(5.9)$ & $-0.000(2.5)$ & $-0.000(3.2)$ & $-0.000(3.1)$ \\
Low education & $-0.184(2.6)$ & $-0.036(0.5)$ & $0.016(0.3)$ & $0.017(0.4)$ & $0.045(1.1)$ \\
Medium education & $-0.204(2.9)$ & $-0.118(1.7)$ & $-0.098(1.8)$ & $-0.054(1.2)$ & $-0.011(0.3)$ \\
High education & $0.539(6.9)$ & $0.466(6.2)$ & $0.446(7.3)$ & $0.523(9.9)$ & $0.531(11.5)$ \\
$N$ & 86,340 & 76,175 & 81,140 & 83,315 & 82,436 \\
\hline
\end{tabular}

Probit regressions for different years (columns). $t$ statistics in parentheses. rank diff from upper $b d$. $(\theta)$ rank difference between the rank of the individual's own wage and the rank of the upper bound for correction, $\theta_{t s i}=0.88-F_{t} f\left(w_{a g} e_{t s i}\right)$ (see Sect. 3). low education intermediate high school degree after ten years, medium education high school degree after at least twelve years or a vocational degree, high education college degree. The regression also includes ten dummies for different occupation categories, 13 dummies for different industries, and ten dummies for the West German states. The reference category involves observations with missing values for education, occupation, industries, and states. The employment spells are weighted by their length

specifies the probability of reporting part-time as a function of the wage position $\theta_{t s i}$ and a vector of characteristics $x_{t s i}$, with

$$
\operatorname{Pr}\left(p t_{t s i}=1 \mid \theta_{t s i}, x_{t s i}\right)=\Phi\left(\alpha_{t}+\beta_{t} \theta_{t s i}+x_{t s i}^{\prime} \gamma_{t}\right)
$$

where $\Phi($.$) is the distribution function of the standard$ normal. The controls contained in $x_{t s i}$ are a second order polynomial for age, dummies for low, medium and high educational attainments, ten dummies for different job categories, 13 dummies for different industries, and ten dummies for the West German states. The reference category for educational categories, job categories, sectors and states includes observations with missing values.

The probit regressions yield the predicted probabilities for a spell to be reported as a part-time spell for the years 2000 to 2012. Since the part-time share increases substantially after 2011, we expect for the vast majority of observations in years $t \leq 2011$ that $\operatorname{Pr}\left(p t_{2012 s i}=1 \mid \theta_{t s i}, x_{t s i}\right)>\operatorname{Pr}\left(p t_{t s i}=1 \mid \theta_{t s i}, x_{t s i}\right)$, i.e. for given spell characteristics $\theta_{t s i}, x_{t s i}$ the predicted part-time probability based on the 2012 regression exceeds the predicted part-time probability based on the regression for the earlier year $t$. This expectation is confirmed in the data. Tables 1 and 2 show the coefficient estimates for the probit regressions for selected years. The results confirm that the part-time probabilities increase in the rank difference $\theta_{t s i}$. For women and men, its effect is highly significant and it tends to be larger in size for the latter.

\subsection{Weights}

In the final step, we calculate the full-time weight $f t_{-}$weight $_{t s i}$, i.e. the reweighting factor, as the ratio of the predicted probabilities of being reported to work fulltime in the year 2012 and the year $\mathrm{t}$

$$
\begin{aligned}
f t_{-} \text {weight }_{t s i} & =\min \left(\frac{\operatorname{Pr}\left(p t_{2012 i}=0 \mid \theta_{t s i}, x_{t s i}\right)}{\operatorname{Pr}\left(p t_{t s i}=0 \mid \theta_{t s i}, x_{t s i}\right)}, 1\right) \\
& =\min \left(\frac{1-\operatorname{Pr}\left(p t_{2012 i}=1 \mid \theta_{t s i}, x_{t s i}\right)}{1-\operatorname{Pr}\left(p t_{t s i}=1 \mid \theta_{t s i}, x_{t s i}\right)}, 1\right) .
\end{aligned}
$$

We censor the weight at one, i.e. the weight is set to one if the ratio exceeds one.

The goal is to downweight observations in year $t$ which given their characteristics have a lower full-time probability in 2012 than the probability of being reported full-time in year $t$. Indeed, the weights prove to be less than one for more than $91 \%$ of spells, which means that $\operatorname{Pr}\left(p t_{2012 s i}=1 \mid \theta_{t s i}, x_{t s i}\right)>\operatorname{Pr}\left(p t_{t s i}=1 \mid \theta_{t s i}, x_{t s i}\right) \quad$ and $f t_{-}$weight $_{t s i}<1$. This corresponds to our assumption that full-time employment is overstated before the 2011 break in the part-time indicator. For the remaining spells, the ratio of the probabilities is larger than or equal to one and we cap the ratio at one, which means that such spells are not reweighted. Based on our understanding of the measurement error in the data, we do not increase the actual sample weight of a spell reported as full-time in the raw data. ${ }^{9}$

Figure 4 provides local linear regression estimates on the relationship between the full-time weights and the rank difference $\theta_{t s i}$ as further plausibility check. For wages close to the upper bound (a rank difference close to zero) the weights are close to one, indicating that the

\footnotetext{
${ }^{9}$ One may wonder as to whether one should also correct spells which are reported as part-time spells and which may in fact be full-time spells. Such a correction would be beyond the scope of our paper. This type of misclassification seems less of an issue given the strong evidence for the bias introduced by part-time spells being reported as full-time spells up to 2010, see Fig. 1 . However, we leave a careful examination of this to future research.
} 
Table 2 Probit regression for part-time spell reported, women

\begin{tabular}{llllll}
\hline & $\mathbf{( 1 )}$ & $\mathbf{( 2 )}$ & $\mathbf{( 3 )}$ & $\mathbf{( 4 )}$ & $\mathbf{( 5 )}$ \\
& $\mathbf{2 0 0 0}$ & $\mathbf{2 0 0 5}$ & $\mathbf{2 0 1 0}$ & $\mathbf{2 0 1 1}$ & $\mathbf{2 0 1 2}$ \\
\hline Rank diff from Upper bd. $(\theta)$ & $2.814(146.8)$ & $2.558(135.2)$ & $2.355(133.6)$ & $2.804(156.4)$ & $3.061(163.4)$ \\
Age & $0.224(45.8)$ & $0.220(46.6)$ & $0.197(44.4)$ & $0.218(49.6)$ & $0.229(52.0)$ \\
Age2 & $-0.002(39.8)$ & $-0.002(40.1)$ & $-0.002(37.3)$ & $-0.002(42.3)$ & $-0.002(44.1)$ \\
Low education & $-0.009(0.2)$ & $0.017(0.3)$ & $0.090(2.0)$ & $0.109(2.6)$ & $0.075(1.9)$ \\
Medium education & $-0.027(0.6)$ & $0.005(0.1)$ & $0.067(1.6)$ & $0.209(5.1)$ & $0.153(4.0)$ \\
High education & $0.382(7.8)$ & $0.341(6.5)$ & $0.327(7.3)$ & $0.511(11.9)$ & $0.497(12.3)$ \\
$N$ & 155,868 & 142,906 & 158,310 & 164,041 & 166,134 \\
\hline
\end{tabular}

Probit regressions for different years (columns). $t$ statistics in parentheses. rank diff from upper $b d$. $(\theta)$ rank difference between the rank of the individual's own wage and the rank of the upper bound for correction, $\theta_{t s i}=0.29-F_{t \_} m\left(\right.$ wage $\left._{t s i}\right)$ (see Sect. 3). low education intermediate high school degree after ten years, medium education high school degree after at least twelve years or a vocational degree, high education college degree. The regression also includes ten dummies for different occupation categories, 13 dummies for different industries, and ten dummies for the West German states. The reference category involves observations with missing values for education, occupation, industries, and states. The employment spells are weighted by their length

2012 part-time probability and the probability of being reported full-time in the observation year are very similar. The weight decreases for a growing rank difference, implying that spells with lower wages are more likely to be misreported. For both genders, the estimates for the weights cover the part of the distribution below the initially defined upper bound-every spell below the 88th and 29th percentile for women and men, respectively, in the total employment sample.

For our subsequent analysis of wage inequality, we weight the spells by the product of the full-time weight in Eq. (2) and the relative length of the spell. The resulting spell weight becomes

$$
\text { weight }_{t s i}=f t_{-} \text {weight }_{t s i} \cdot \frac{\text { length }_{t s i}}{n d a y s_{t}},
$$

where length $h_{t s i}$ denotes the length of the spell in days and ndays $_{t}$ the total number of days in year t, 365 or 366, respectively.

\section{Trends in wage inequality before and after the correction}

We now investigate whether the paramount evidence reported in the literature for rising wage inequality until 2010 among full-timers is robust against the misreporting of low-wage part-time employment in the raw SIAB data as full-time employment during that time period. Doing so, we revisit the evidence reported in Möller (2016) showing that correcting the data before 2011 does not qualitatively change the finding of a strong rise in wage inequality among full-timers until $2010 .^{10}$

\footnotetext{
${ }^{10}$ Recall that our analysis is restricted to West Germany whereas Möller (2016) also provides evidence for East Germany.
}

Downweighting the full-time employment spells up to 2011 mainly affects wages in the lower part of the distribution but still also changes higher wage percentiles. This is because a reduction in the weighted shares of workers with low wages mechanically increases all percentiles up the wage distribution, an effect which goes even beyond the percentiles above the upper bounds used for the correction. However, this increase in all percentiles is not uniform across the wage distribution-in fact the increase becomes smaller further up the distribution. Thus, the correction reduces the level of wage inequality and it may possibly affect the estimated trend of wage inequality.

Figure 5 shows the trends of original and corrected percentiles of the wage (in logs) distribution for women and men from 2000 onward. The figure shows that the upward correction is stronger in the lower tail of the wage distribution and, holding the wage level constant, the correction is stronger for women than for men. The correction for men at the median and the 25 th percentile is small but still visible and it becomes sizeable at the 5 th percentile for men. For women, the correction is sizeable even at the median and it grows further moving down the wage distribution. To give some numbers for 2010, the upward correction for women is $0.12 \log$-points ( $3 \%$ of the real log wage) at the 25 th percentile and $0.18 \log$ points $(5 \%)$ at the 5 th percentile. For men, it is $0.01 \log$-points $(0.3 \%)$ at the 25 th percentile and $0.04 \log$ points $(1.0 \%)$ at the 5 th percentile. As intended, the correction smoothes the discontinuous development of the lower percentiles between 2010 and 2012. Even though the correction is stronger before 2011, the findings indicate a need for 


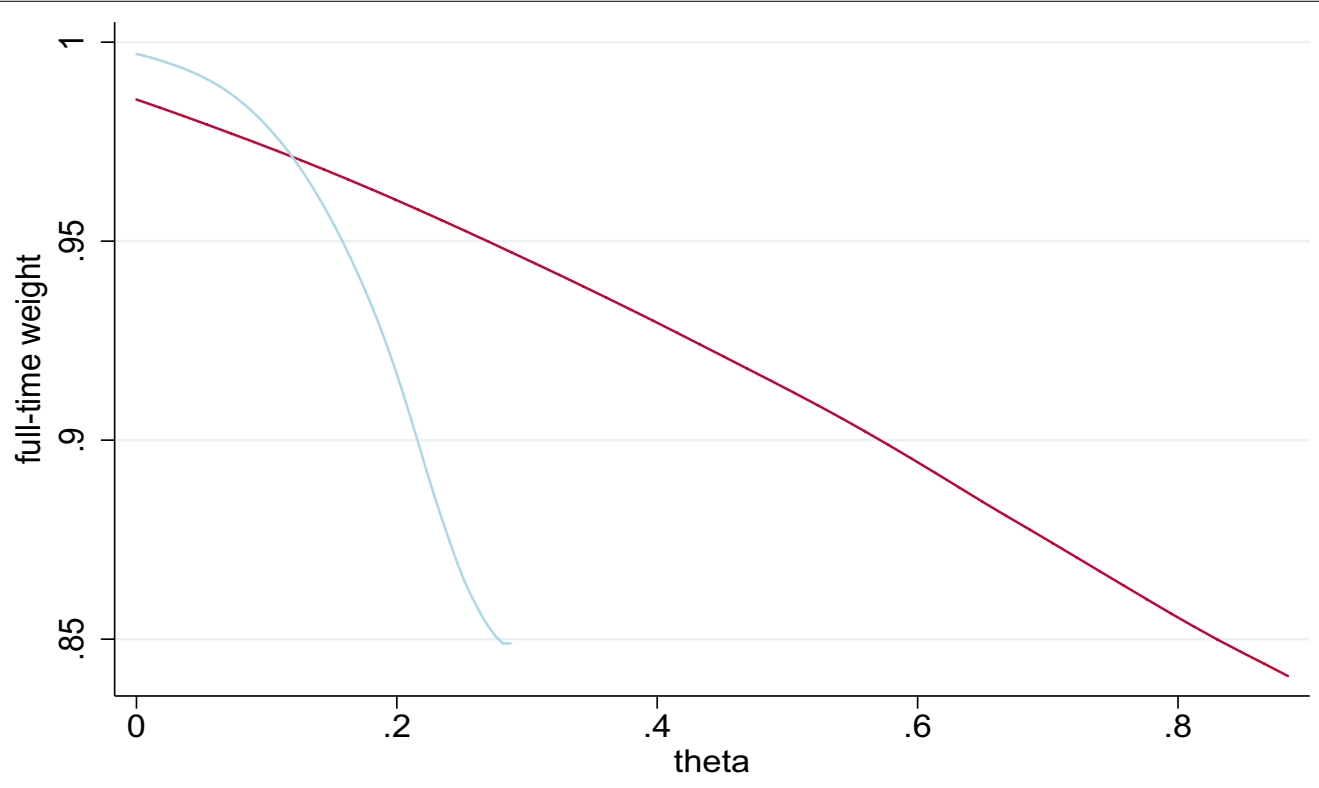

Women

Men

Fig. 4 Local linear regression for the full-time weight. Estimates for a local linear regression of the full-time weight on the rank difference $\theta$. For individual percentile ranks close to the upper bound until we correct, $\theta$ approaches zero. $\theta$ is the larger the lower the rank in the wage distribution. For women and men, only the part below the respective upper bound, 88th and 29 percentile, is considered for the correction

correction in 2011, the year of the break in the parttime indicator. ${ }^{11}$

Clearly, the correction reduces the gap between different percentiles, which means that it reduces the measured wage inequality until 2011 compared to the original data. Despite this, the graphical evidence in Fig. 5 suggests that the corrected percentiles and the percentiles based on the raw data seem to show quite similar trends up to 2010. Figures 6 and 7 illustrate both points, showing time trends for the difference between different percentiles of log wages. Wage inequality for females is higher in the lower half of the distribution, because the difference between the 50th and 20th percentile is larger than the one between the 80th and the 50th percentile. The full-time correction has its strongest effect on the former as shown in Fig. 6. Based on Fig. 7, the correction for men is small in comparison and the difference

\footnotetext{
${ }^{11}$ However, the implied trends of wage percentiles for the corrected data are not smooth in 2011. This would deserve further investigation based on the raw social security records, which we do not have access to. This nonsmooth trends in 2011 may reflect the uncertainty involved with the change in the reporting procedure because the SIAB7514 data already involves an imputation of the reported part-time status in 2011 for observations with missing data, see Ludsteck and Thomsen (2016). Further note that it is difficult to pin down the trend in 2011 in light of the likely trend break in the evolution of wage inequality during the years 2010/2011 - based on the results reported in Möller (2016) and in this paper.
}

in the upper half of the distribution is larger than in the lower half. Figures 8 and 9 provide a more detailed view on wage trends by showing the cumulative growth since 2000 (measured as log differences) of wages at different percentiles.

In contrast to the period until 2010, the trend in wage inequality between 2010 and 2014 is strongly affected by our correction. Based on the raw uncorrected data, one would be inclined to conclude that wage inequality among women decreases strongly between 2010 and 2014 (e.g. the 80-20 gap decreases by about $10 \log$ points, Figs. 6 and 8 to the left) and it levels off for men (Figs. 7 and 9 to the left). After the correction, wage inequality for both genders levels off and, especially in the lower part of the wage distribution, there is less real wage growth after 2010 (Figs. 8 and 9 to the right).

As final part of our empirical analysis, we investigate the age dimension of wage inequality. Antonczyk et al. (2018) find that an important aspect of the rise of wage inequality among men was that wage differences between older and younger workers increase strongly until 2004 and that real wages fell strongly for younger workers, especially in the lower part of wage distribution. Against this backdrop, we contrast workers aged 25 to 34 and those aged 35 to 55 . Specifically, we investigate whether wage trends for younger workers at the bottom of the wage distribution continued to fall strongly after 2004 


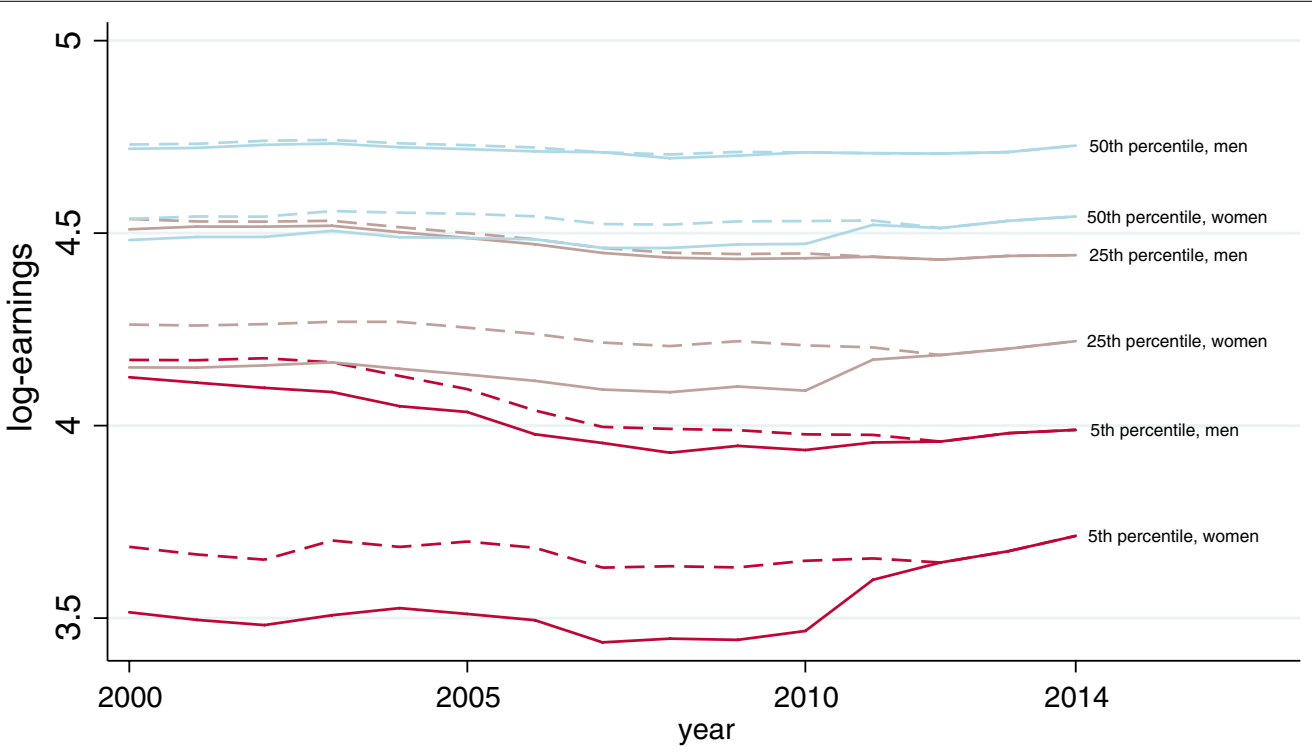

Solid Line: Raw data, weighted by the length of spell Dashed Line: Part-time corrected

Fig. 5 Percentiles of log-wage distribution, full-time, raw and corrected. Percentiles of the log-wage distribution for full-time employees, inflation adjusted, only former West-Germany (without Berlin), only employees aged 25 to 55, without marginally employed and apprentices, weighted by the length of employment spells

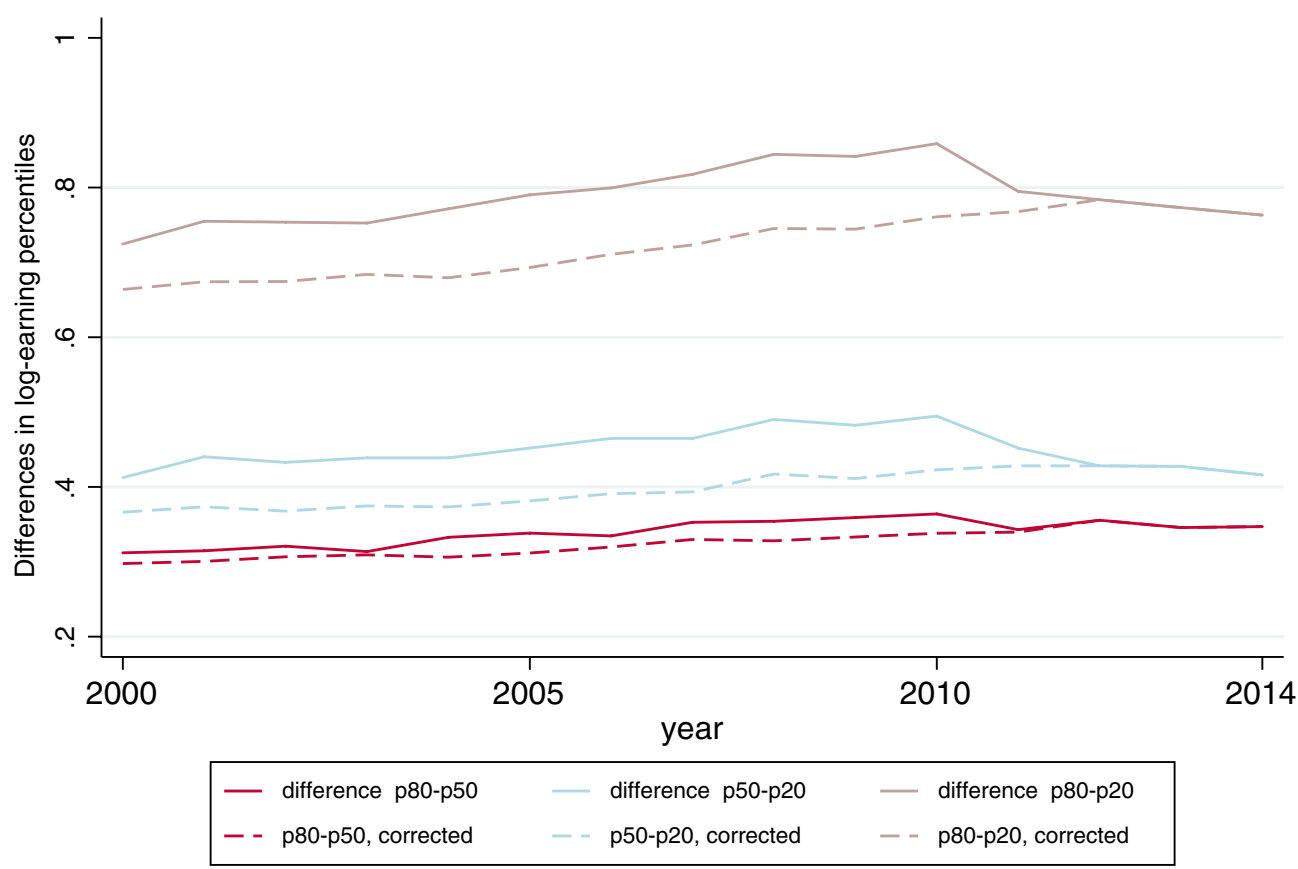

Fig. 6 Percentile differences of log-wage distribution for women, full-time, raw and corrected. Differences between the 80th and 50th, 50th and 20th and 80th and 20th percentile of the log-wage distribution for full-time employees, inflation adjusted, only former West-Germany (without Berlin), only employees aged 25 to 55, without marginally employed and apprentices, weighted by the length of employment spells 


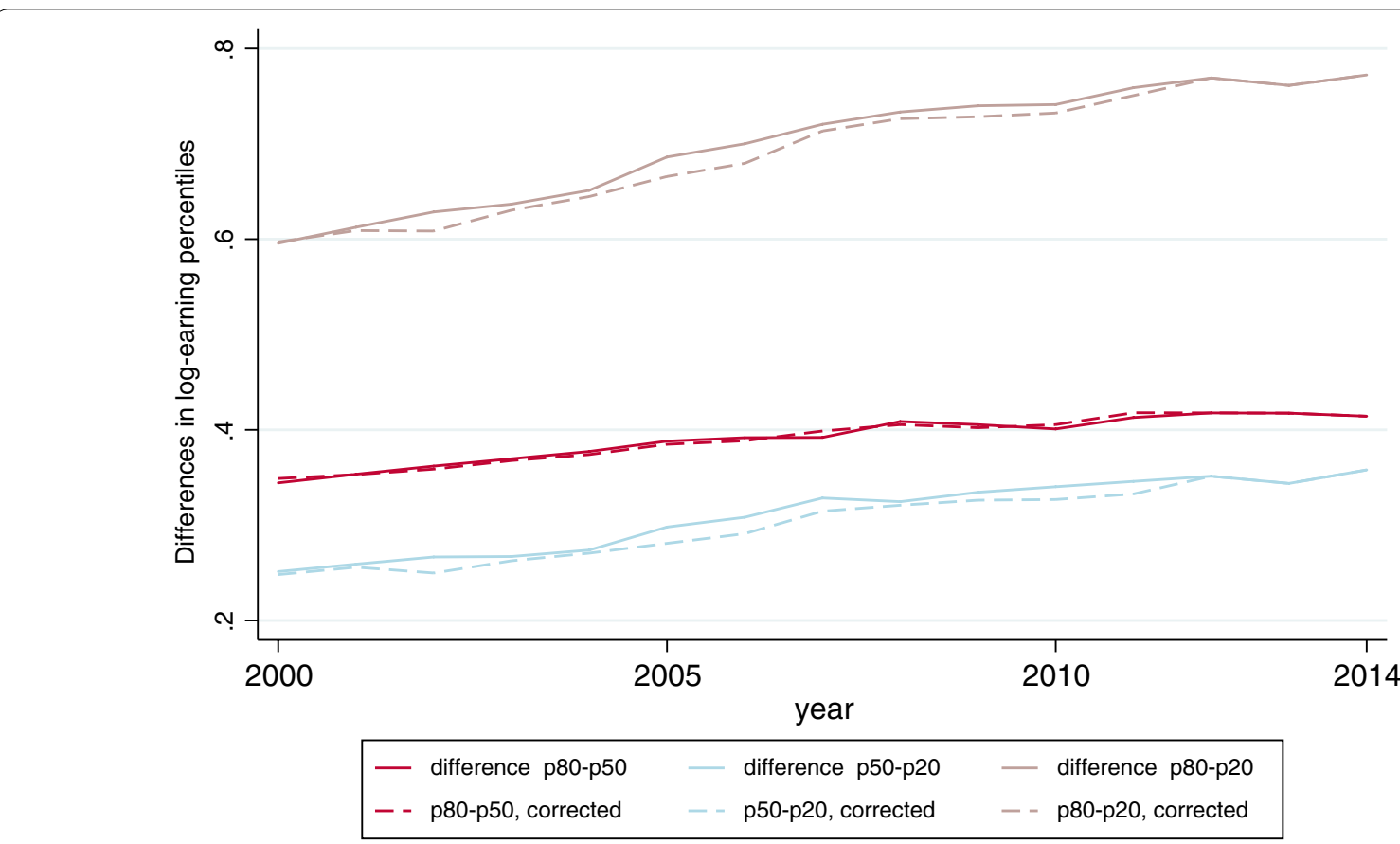

Fig. 7 Percentile differences of log-wage distribution for men, full-time, raw and corrected. Differences between the 80th and 50th, 50th and 20th and 80th and 20th percentile of the log-wage distribution for full-time employees, inflation adjusted, only former West-Germany (without Berlin), only employees aged 25 to 55, without marginally employed and apprentices, weighted by the length of employment spells

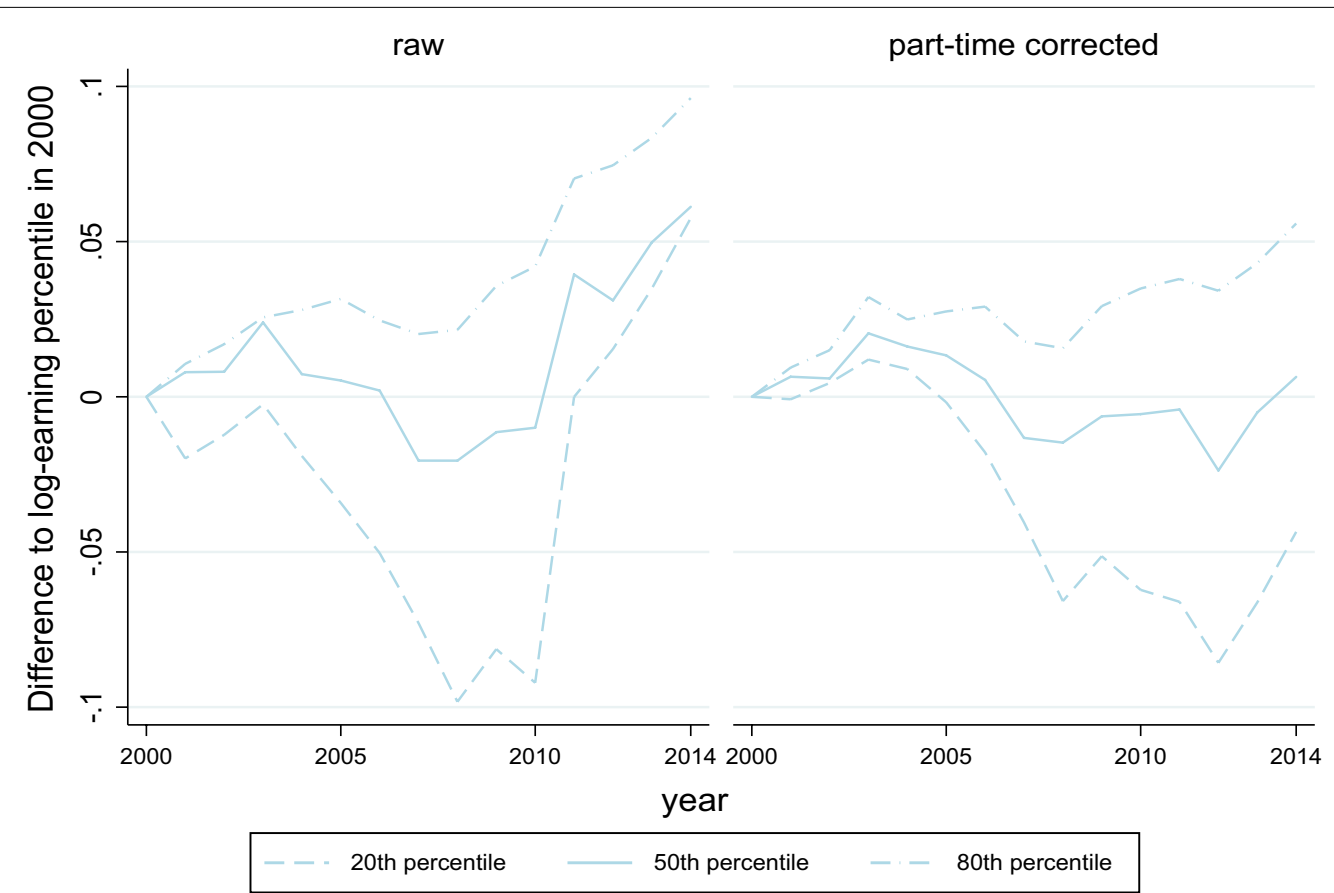

Fig. 8 Cumulative real wage growth for women, full-time. Differences in log real wages over time, indexed to 0 in year 2000, only former West-Germany (without Berlin), only employees aged 25 to 55, without marginally employed and apprentices, weighted by the length of employment spells 


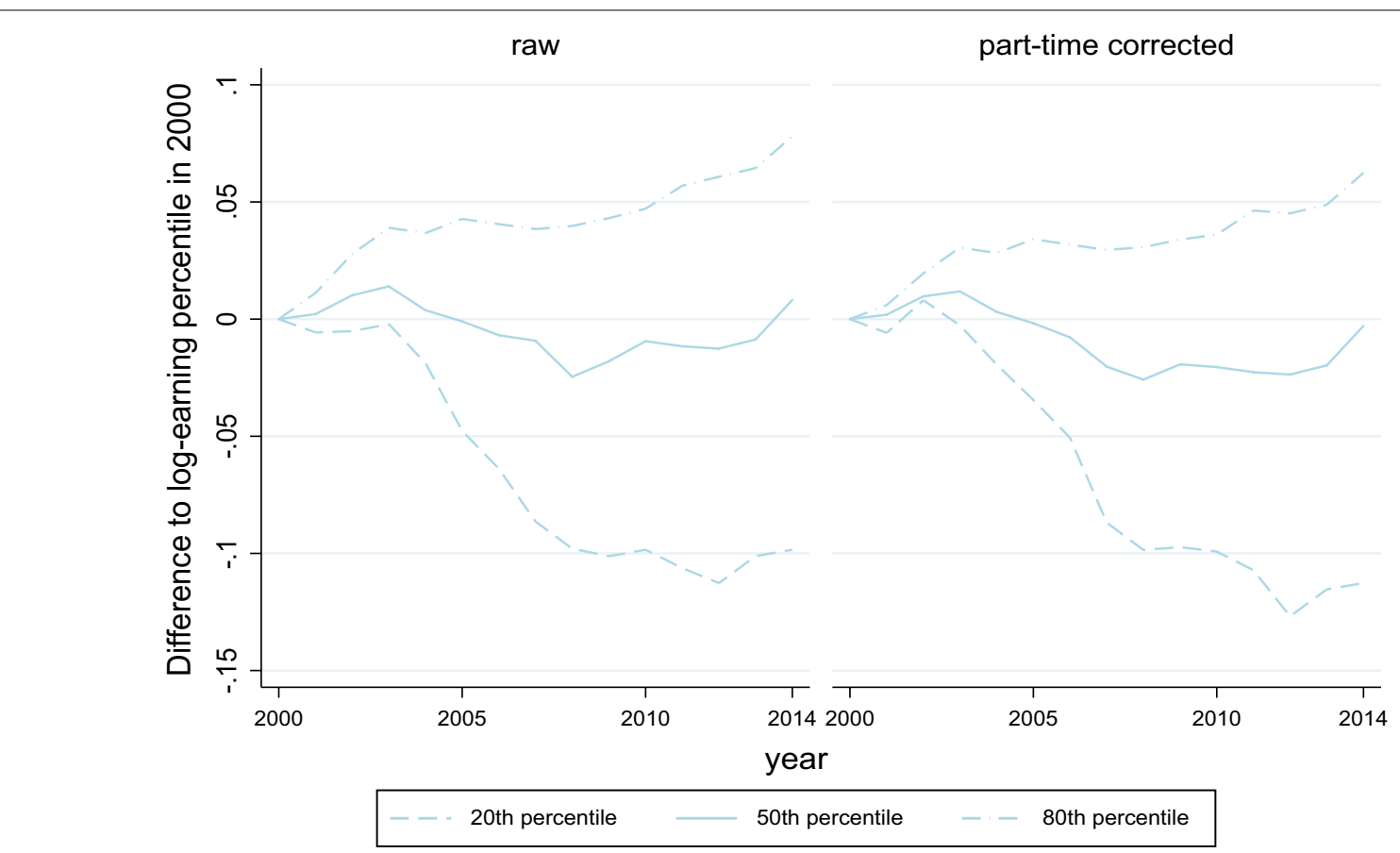

Fig. 9 Cumulative real wage growth for men, full-time. Differences in log real wages over time, indexed to 0 in year 2000, only former West-Germany (without Berlin), only employees aged 25 to 55, without marginally employed and apprentices, weighted by the length of employment spells

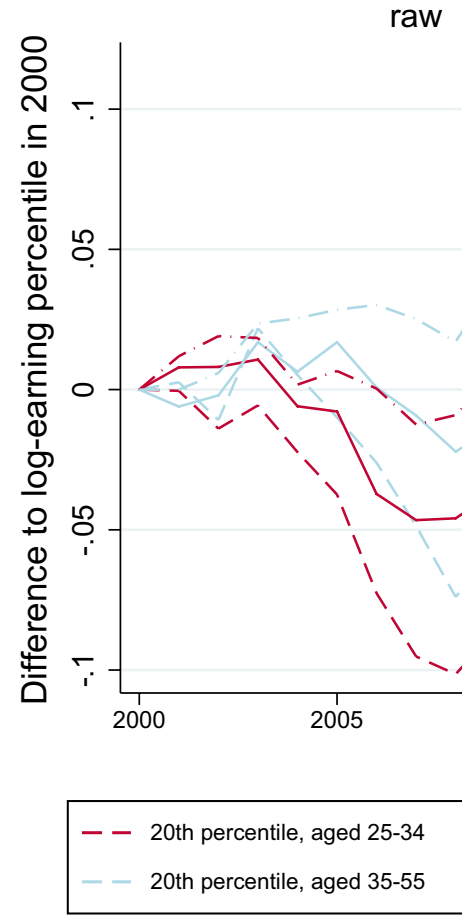

raw

\section{part-time corrected}

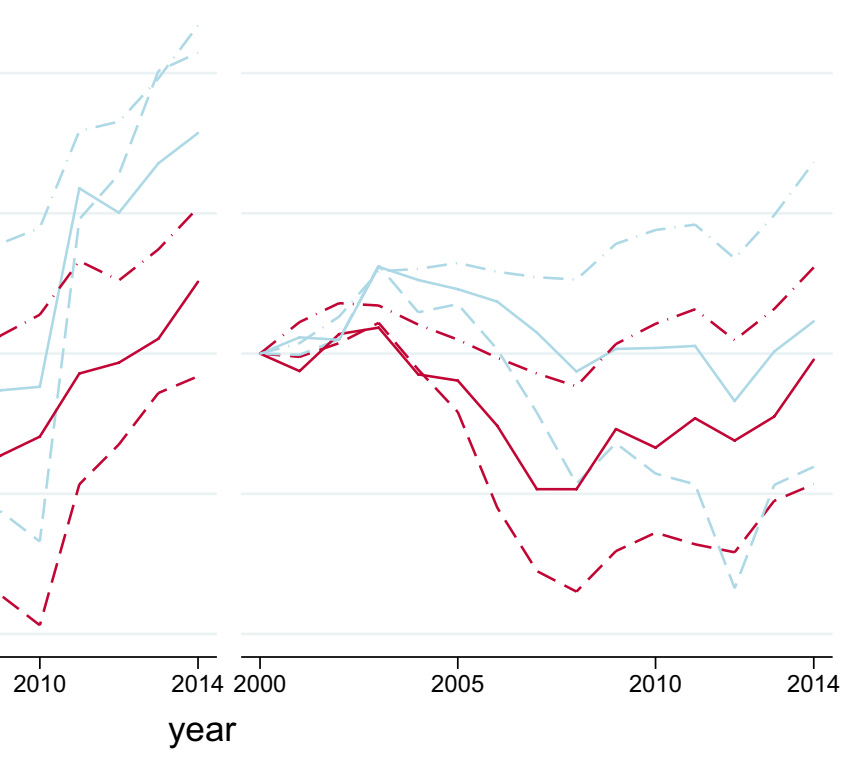

\section{0th percentile, aged 25-34}

- - 80th percentile, aged 25-34

50th percentile, aged $35-55$

- - 80th percentile, aged 35-55

Fig. 10 Cumulative real wage growth for women by age groups, full-time. Differences in log real wages over time, indexed to 0 in year 2000 , only former West-Germany (without Berlin), only employees aged 25 to 55, without marginally employed and apprentices, weighted by the length of employment spells 


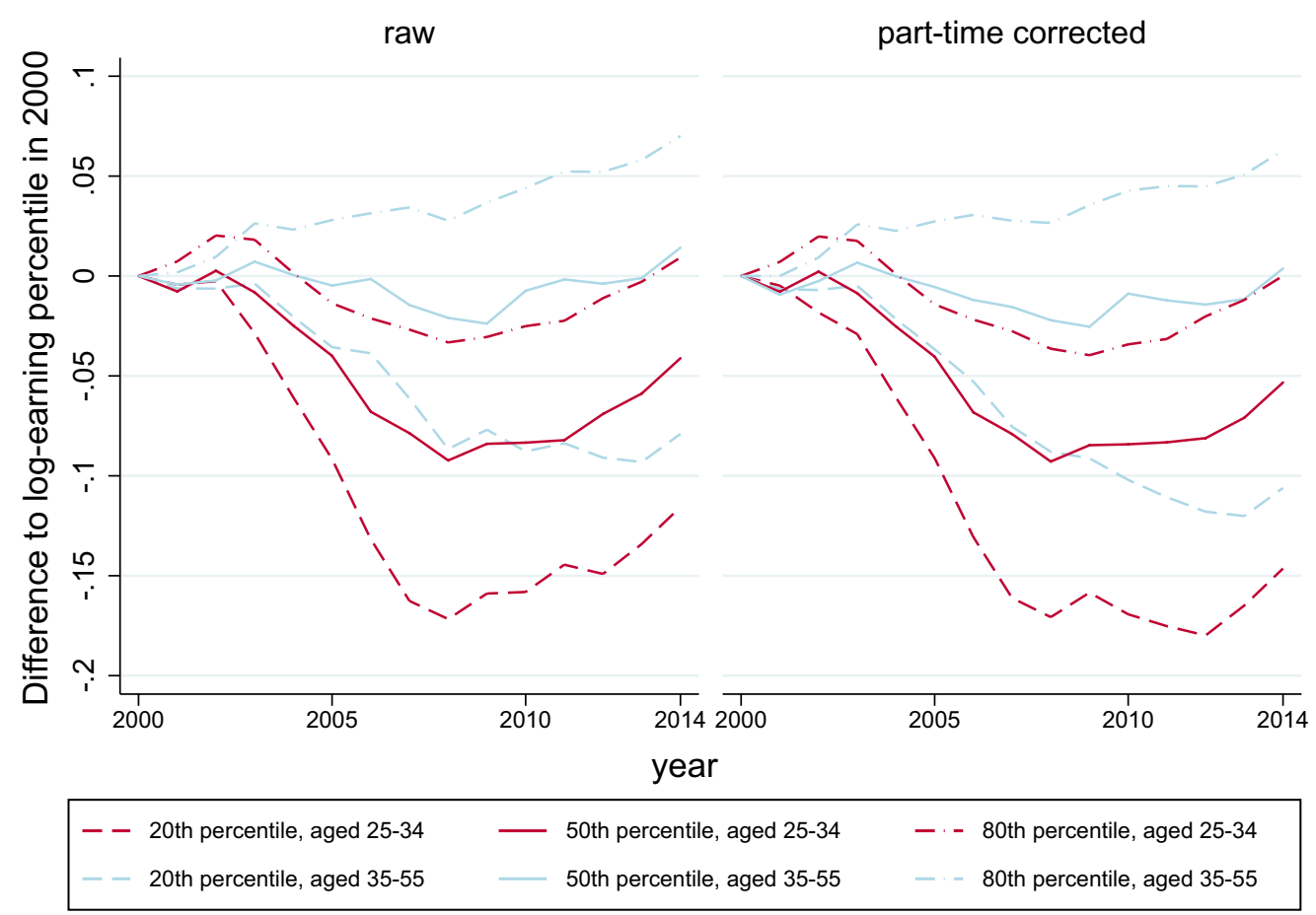

Fig. 11 Cumulative real wage growth for men by age groups, full-time. Differences in log real wages over time, indexed to 0 in year 2000 , only former West-Germany (without Berlin), only employees aged 25 to 55, without marginally employed and apprentices, weighted by the length of employment spells

and whether there was a reversal after 2010, while checking whether key results change after applying our correction. Figures 10 and 11 show the wage trends by age groups and gender, both based on the raw data and the corrected data.

Our findings show that cumulative wage growth at all percentiles is lower for younger workers than for older workers and that wage inequality within wage groups grows strongly over time (because wage growth at lower percentiles is lower than at higher percentiles). The effects of the correction are similar to what has been discussed above for the overall wage distribution. The correction reduces the wage growth after 2010, especially in the lower tail of the wage distribution and for women. The key findings are that there was a very strong fall of real wages for young workers until 2010, especially at lower percentiles with the 20th percentile falling by about $10 \log$ points for women and $17 \log$ points for men. After 2010 , there is a modest recovery of wages for both men and women except for the 20th percentiles for men in both age groups and the 20th percentile for older women. Incidentally, wages at the 20th percentile for young women grow in parallel to the other percentiles in that group. Our findings confirm the strong decline of real wages for young workers at low percentiles [as stressed by Antonczyk et al. (2018)] for men during a longer time period (until 2010). Further, there is little indication of a recovery after 2010. Clearly, these findings would deserve more scrutiny, which is, however, beyond the scope of this paper.

\section{Conclusions}

The Sample of Integrated Labor Market Biographies (SIAB) are based on German social security records which involve an indicator for part-time or full-time work. These data are widely used to analyze trends in wage inequality among full-time workers. The reporting procedure for the part-time indicator changed in 2011 with dramatic consequences on the share of reported part-time workers. This paper develops a refined correction procedure for this break and investigates the robustness of previous findings on the evolution of wage inequality in Germany. We argue that the full adjustment to the new reporting procedure was completed only in 2012 and therefore we also apply our correction approach to the data for 2011.

Our correction approach involves estimating the probability of being reported to work part-time as a function of the rank difference in the wage distribution among all employees (full-timers and part-timers) for all years 2000 
to 2012. The full-time employment data before 2012 is then reweighted using inverse probability reweighting based on the estimated propensity scores. This approach detects and downweights observations which are likely to be misreported as full-time, which results in a continuous upward correction of low wage percentiles among full-timers. We plan to make the correction procedure available to all users of the SIAB data.

Using our correction, the paper confirms that the rise in wage inequality among full-time workers in West Germany until 2010 is not a spurious consequence of the misreporting of working time. Furthermore, based on our corrected data, we find that the fall in real wages among full-timers during the 2000s was strongest among young workers and there is in fact a trend reversal after 2010, as already observed by Möller (2016). While the raw wage data show strong wage growth for women after 2010, the correction shows that most of this growth is spurious. In fact, based on the corrected data, wage trends between 2010 and 2014 have contributed little to reverse the strong increase in wage inequality until 2010, a findings which holds in particular for low-wage earners among men. On a methodological note, our findings show the importance of correcting for the break in the part-time indicator when analyzing wage trends.

Future research should determine whether further key results in the literature on trends in wage inequality for West Germany (see e.g. Card et al. 2013; Biewen et al. 2018) are robust when applied to the corrected data. In addition, it will be of great interest to extend the analysis beyond the year 2014. From a methodological perspective, this will allow to estimate longer term trends for data based on the new reporting procedure fully in place since 2012. From a substantive perspective, it will be interesting to investigate the impact of the long labor market boom with continuously falling unemployment since 2010 and the introduction of the national minimum wage in 2015 on wage trends and wage inequality.

\section{Supplementary information}

Supplementary information accompanies this paper at https://doi. org/10.1186/s12651-019-0265-0.

Additional file 1. Readme file on data preparation.

Additional file 2. Stata code used for data data preparation.

\section{Acknowledgements}

We are very grateful to Johannes Ludsteck, Joachim Möller, Dana Müller, and Phillip vom Berge for helpful discussions.

\section{Authors' contributions}

$\mathrm{BF}$ and $\mathrm{AS}$ conceived the study. BF developed the reweighting procedure and AS wrote the programs and analyzed the data. Both authors decided about the different steps of the empirical analysis and wrote the manuscript. Both authors read and approved the final manuscript.

\section{Funding}

This paper is part of the project "Female Employment Patterns, Fertility, Labor Market Reforms, and Social Norms: A Dynamic Treatment Approach", DFG project number: Fl 692/14-1 and PA 2536/1-1. Financial support by the DFG is gratefully acknowledged.

\section{Availability of data and materials}

All calculations in this paper were done based in the SIAB7514, which is available at the Research data centre (RDC) of the Institute for Employment Research (IAB) and the Federal Employment Agency in Nuremberg. The SIAB7514 is described by Ganzer et al. (2017). Due to data confidentiality restrictions the data cannot be made available in a repository but researcher can access the data through the RDC. A description of the data preparation and the STATA code used are available after publication of this paper (Additional files 1, 2).

\section{Competing interests}

The authors declare that they have no competing interests.

\section{Author details}

${ }^{1}$ IAB, Humboldt University Berlin, IFS, CESifo, IZA, ROA, Nuremberg, Germany. ${ }^{2}$ Humboldt University Berlin, Berlin, Germany. ${ }^{3}$ IAB (Institute for Employment Research), Regensburger Straße 100, 90478 Nuremberg, Germany.

Received: 19 July 2019 Accepted: 24 October 2019

Published online: 02 January 2020

\section{References}

Antonczyk, D., DeLeire, T., Fitzenberger, B.: Polarization and rising wage inequality: comparing the U.S. and Germany. Econometrics $\mathbf{6}(2), 20$ (2018)

Bertat, T., Dundler, A., Grimm, C., Kiewitt, J., Schomaker, C., Schridde, H., Zemann, C.: Neue Erhebungsinhalte "Arbeitszeit" "ausgeübte Tätigkeit" sowie "Schul- und Berufsabschluss" in der Beschäftigungsstatistik. Methodenbericht der Statistik der BA (2013)

Biewen, M., Seckler, M.: Unions, internationalization, tasks, firms, and worker characteristics: a detailed decomposition analysis of rising wage inequality in Germany. J. Econ. Inequal. (2019). https://doi.org/10.1007/s1088 8-019-09422-w

Biewen, M., Fitzenberger, B., de Lazzer, J.: The role of employment interruptions and part-time work for the rise in wage inequality. IZA J. Lab. Econ. 7(1), 10 (2018)

Bundesagentur für Arbeit: Schlüsselverzeichnis für die Angaben zur Tätigkeit, Ausgabe 2010. Meldeverfahren zur Sozialversicherung (2019)

Card, D., Heining, J., Kline, P.: Workplace heterogeneity and the rise of West German wage inequality. Q. J. Econ. 128(3), 967-1015 (2013)

Dustmann, C., Ludsteck, J., Schönberg, U.: Revisiting the German wage structure. Q. J. Econ. 124(2), 843-881 (2009)

Frodermann, C., Müller, D., Abraham, M.: Determinanten des Wiedereinstiegs von Müttern in den Arbeitsmarkt in Vollzeit oder Teilzeit. KZfSS Kölner Zeitschrift für Soziologie und Sozialpsychologie 65(4), 645-668 (2013)

Ganzer, A., Schmucker, A., vom Berge, P., Wurdack, A.: Sample of integrated labour market biographies_regional file 1975-2014 (SIAB7514). FDZDatenreport, IAB Nürnberg, 1/2017 (2017)

Ludsteck, J., Thomsen, U.: Imputation of the working time information for the employment register data. FDZ-Methodenreport, IAB Nürnberg, 1/2016 (2016)

Möller, J.: Lohnungleichheit—Gibt es eine Trendwende? Wirtschaftsdienst 96(1), 38-44 (2016)

Statistisches Bundesamt:. Verbraucherpreisindizes für Deutschland. Jahresbericht 2017 (2018)

\section{Publisher's Note}

Springer Nature remains neutral with regard to jurisdictional claims in published maps and institutional affiliations. 